EUROPEAN JOURNAL OF PURE AND APPLIED MATHEMATICS

Vol. 15, No. 1, 2022, 47-63

ISSN 1307-5543 - ejpam.com

Published by New York Business Global

\title{
Boundedness of non regular pseudo-differential operators on variable exponent Triebel-Lizorkin-Morrey spaces
}

\author{
Mohamed Congo $^{1, *}$, Marie Françoise Ouedraogo ${ }^{1}$ \\ ${ }^{1}$ Laboratoire de Théorie des Nombres, Algèbre, Géométrie Algébrique, Topologie Algébrique \\ et Applications(TN-AGATA). UFR Sciences Exactes et Appliquées/ Université Joseph \\ KI-ZERBO, 03 BP 7021 Ouaga 03, Ouagadougou, Burkina Faso
}

\begin{abstract}
In this paper, we study the boundedness of non regular pseudo-differential operators on variable exponent Besov-Morrey spaces $\mathcal{E}_{p(\cdot), u(\cdot), q(\cdot)}^{s(\cdot)}$ with symbols $a(x, \xi)$ belonging to $C_{*}^{\ell} S_{1, \delta}^{m}$. For these symbols $x$-regularity is measured in Hölder-Zygmund spaces.
\end{abstract}

2020 Mathematics Subject Classifications: 42B35,46E30,35S05

Key Words and Phrases: Pseudo-differential operators, Non regular symbols, Variable exponent Triebel-Lizorkin-Morrey spaces

\section{Introduction}

Pseudo-differential calculus is a well-established tool for the analysis of partial differential equations, especially non-linear ones. Indeed, in [16] one can find many applications of the calculus of non regular pseudo-differential operators to non-linear differential equations. The boundedness of these operators has been extensively addressed in several works. For boundedness on Lebesgue spaces, Besov spaces, Triebel-Lizorkin spaces and Sobolev spaces, we refer to [2], [6], [12] and [13].

The boundedness of pseudo-differential operators in Triebel-Lizorkin-Morrey spacses with constant exponents denoted $\mathcal{E}_{p, u, q}^{s}$ was studied by Yoshihiro Sawano in [15].

Our focus in this paper concerns the boundedness of pseudo-differential operators on Triebel-Lizorkin-Morrey spaces with variable exponents denoted $\mathcal{E}_{p(\cdot), u(\cdot), q(\cdot)}^{s(\cdot)}($ see $[4])$ with symbols in the class $C_{*}^{\ell} S_{1, \delta}^{m}$.

The results of this paper are certainly relevant because they generalize those of [15].

Our approach is as follows. To treat the boundedness of these operators with non-regular symbols belonging to $C_{*}^{\ell} S_{1, \delta}^{m}$ we use elementary symbols as it was done in [2], [12], [14]

*Corresponding author.

DOI: https://doi.org/10.29020/nybg.ejpam.v15i1.4200

Email addresses: mohamed.congo@yahoo.fr (M. Congo), omfrancoise@yahoo.fr (M F. Ouedraogo) 
and [15].

Indeed, the symbol reduction method, due to Coifman and Meyer[6], makes it possible to be limited to symbols $a(x, \xi) \in C_{*}^{\ell} S_{1, \delta}^{m}$ of the form $a(x, \xi)=\sum_{j \geq 0} \sigma_{j}(x) \psi_{j}(\xi)$ (see [14] and [2]). Then, we rewrite the symbol as a sum of three parts, a "low-high", a "high-high", and a "high-low" part. Thus, the operator $a(x, D)$ with symbol $a$ can be resolved into three operators $a_{1}(x, D), a_{2}(x, D)$ and $a_{3}(x, D)$ with symbols $a_{1}, a_{2}$ and $a_{3}$. Now it remains to study the boundedness of each elementary operators.

We structure this paper in $\mathbf{4}$ sections as follows. In Section $\mathbf{2}$ we give the preliminaries, where we recall the definitions of Morrey spaces and Besov-Morrey spaces with variable exponents. In Section 3, we recall necessary tools for the proofs of the lemmas and the main result that we give in Section 4.

\section{Preliminaries}

We denote by $\mathbb{R}^{n}$ the $n$-dimensional real Euclidean space, $\mathbb{N}$ the collection of all natural numbers and $\mathbb{N}_{0}=\mathbb{N} \cup\{0\}$. $\mathbb{Z}$ stands for the set of all integer numbers. We write $B(x, r)$ for the open ball in $\mathbb{R}^{n}$ centered at $x \in \mathbb{R}^{n}$ with radius $r>0$. We use $c$ as a generic positive constant, i.e. a constant whose value may change with each appearance. The expression $f \lesssim g$ means that $f \leq c g$ for some independent constant $c$, and $f \approx g$ means $f \lesssim g \lesssim f$. Throughout the paper we denote by $\mathcal{M}\left(\mathbb{R}^{n}\right)$ the family of all complex or extended real-valued measurable functions on $\mathbb{R}^{n}$.

By $\operatorname{supp} f$ we denote the support of the function $f$, i.e., the closure of its non-zero set. If $E \subset \mathbb{R}^{n}$ is a measurable set, then $\chi_{E}$ denotes its characteristic function.

We denote by $\mathcal{S}\left(\mathbb{R}^{n}\right)$ the set of all Schwartz functions on $\mathbb{R}^{n}$. We denote by

$\mathcal{S}^{\prime}=\mathcal{S}^{\prime}\left(\mathbb{R}^{n}\right)$ the dual space of all tempered distributions on $\mathbb{R}^{n}$. The Fourier transform of a tempered distribution $f$ is denoted by $\mathcal{F} f$ or $\hat{f}$ while its inverse transform is denoted by $\mathcal{F}^{-1} f$ or $\check{f}$.

\subsection{Variable exponents}

For more information on the results of this paragraph, see [11] and [7].

- By $\mathcal{P}\left(\mathbb{R}^{n}\right)$ we denote the set of all measurable functions $p: \mathbb{R}^{n} \rightarrow(0,+\infty]$ (called variable exponents) which are essentially bounded away from zero. We denote $p_{\mathbb{R}^{n}}^{+}:=\operatorname{ess} \sup _{\mathbb{R}^{n}} p(x)$ and $p_{\mathbb{R}^{n}}^{-}:=\operatorname{ess} \inf _{\mathbb{R}^{n}} p(x)$; we abbreviate $p^{+}=p_{\mathbb{R}^{n}}^{+}$and $p^{-}=p_{\mathbb{R}^{n}}^{-}$.

- The function $\phi_{p}$ is defined as follows:

$$
\phi_{p(x)}(t)= \begin{cases}t^{p(x)} & \text { if } p(x) \in(0,+\infty) \\ 0 & \text { if } p(x)=+\infty \text { and } t \in[0,1] \\ +\infty & \text { if } p(x)=+\infty \text { and } t \in(1,+\infty]\end{cases}
$$

The variable exponent modular associated to $p(\cdot)$ is defined by

$$
\varrho_{p(\cdot)}(f):=\int_{\mathbb{R}^{n}} \phi_{p(x)}(|f(x)|) \mathrm{d} x .
$$


The variable exponent Lebesgue space $L_{p(\cdot)}:=L_{p(\cdot)}\left(\mathbb{R}^{n}\right)$ is the family of (equivalence classes of functions $f \in \mathcal{M}\left(\mathbb{R}^{n}\right)$ such that $\varrho_{p(\cdot)}(f / \lambda)$ is finite for some $\lambda>0$.

$L_{p(\cdot)}$ is a quasi-Banach space equipped with the quasinorm

$$
\|f\|_{p(\cdot)}:=\inf \left\{\mu>0: \varrho_{p(\cdot)}\left(\frac{1}{\mu} f\right) \leq 1\right\} .
$$

- We say that a continuous function $g: \mathbb{R}^{n} \rightarrow \mathbb{R}$ is locally log-Hölder continuous, abbreviated $g \in C_{l o c}^{\log }\left(\mathbb{R}^{n}\right)$, if there exists $c_{l o g}(g) \geq 0$ such that

$$
|g(x)-g(y)| \leq \frac{c_{\log }(g)}{\log (\mathrm{e}+1 /|x-y|)} \quad \text { for all } x, y \in \mathbb{R}^{n} .
$$

The function $g: \mathbb{R}^{n} \rightarrow \mathbb{R}$ is said to be globally log-Hölder continuous, abbreviated $g \in C^{\log }\left(\mathbb{R}^{n}\right)$, if it is locally log-Hölder continuous and there exists $g_{\infty} \in \mathbb{R}$ and $c_{\infty}(g) \geq 0$ such that

$$
\left|g(x)-g_{\infty}\right| \leq \frac{c_{\infty}(g)}{\log (\mathrm{e}+|x|)} \quad \text { for all } x \in \mathbb{R}^{n}
$$

We write $g \in \mathcal{P}^{\log }\left(\mathbb{R}^{n}\right)$ if $0<g^{-} \leq g(x) \leq g^{+} \leq+\infty$ with $\frac{1}{g} \in C^{\log }\left(\mathbb{R}^{n}\right)$.

We define $\frac{1}{g_{\infty}}:=\lim _{|x| \rightarrow+\infty} \frac{1}{g(x)}$ and we use the convention $\frac{1}{\infty}=0$.

\subsection{Variable exponent Triebel-Lizorkin-Morrey spaces}

We refer to the papers [4], [18], [3], [5], [17] and [9], for further results on TriebelLizorkin-Morrey spaces and variable exponent Triebel-Lizorkin-Morrey spaces.

\section{- Morrey spaces}

Definition 1. For $p, u \in \mathcal{P}\left(\mathbb{R}^{n}\right)$ with $0<p^{-} \leq p(x) \leq u(x) \leq+\infty$, the variable exponent Morrey space $M_{p(\cdot), u(\cdot)}:=M_{p(\cdot), u(\cdot)}\left(\mathbb{R}^{n}\right)$ consists of all functions $f \in \mathcal{M}\left(\mathbb{R}^{n}\right)$ with finite quasinorm

$$
\|f\|_{M_{p(\cdot), u(\cdot)}}:=\sup _{x \in \mathbb{R}^{n}, r>0} r^{\frac{n}{u(x)}-\frac{n}{p(x)}}\left\|f_{\chi_{B(x . r)}}\right\|_{L_{p(\cdot)}} .
$$

By the definition of the $L_{p(\cdot)}$ quasinorm, (2) can also be written as

$$
\|f\|_{M_{p(\cdot), u(\cdot)}}:=\sup _{x \in \mathbb{R}^{n}, r>0} \inf \left\{\lambda>0: \varrho\left(r^{\frac{n}{u(x)}-\frac{n}{p(x)} \frac{f}{\lambda}} \chi_{B(x . r)}\right) \leq 1\right\} .
$$

Definition 2. Let $p, q, u \in \mathcal{P}\left(\mathbb{R}^{n}\right)$ with $p(x) \leq u(x)$. The mixed space $M_{p(\cdot), u(\cdot)}\left(\ell_{q(\cdot)}\right)$ consists of all sequences $\left(f_{\nu}\right)_{\nu} \subset \mathcal{M}\left(\mathbb{R}^{n}\right)$ such that,

$$
\left\|\left(f_{\nu}\right)_{\nu}\right\|_{M_{p(\cdot), u(\cdot)}\left(\ell_{q(\cdot)}\right)}:=\left\|\left(\sum_{\nu=0}^{+\infty}\left|f_{\nu}(\cdot)\right|^{q(\cdot)}\right)^{1 / q(\cdot)}\right\|_{M_{p(\cdot), u(\cdot)}}<+\infty .
$$


Remark 1. [4] Note that $\|\cdot\|_{M_{p(\cdot), u(\cdot)}\left(\ell_{q(\cdot)}\right)}$ defined a quasinorm on $M_{p(\cdot), u(\cdot)}\left(\ell_{q(\cdot)}\right)$. It is a norm when $\min \left(p^{-}, q^{-}\right) \geq 1$.

Proposition 1. Let $f$ and $g$ be two measurable functions with $0 \leq f(x) \leq g(x)$ for a.e. $x \in \mathbb{R}^{n}$. Then it holds

$$
\|f\|_{M_{p(\cdot), u(\cdot)}\left(\ell_{q(\cdot)}\right)} \leq\|g\|_{M_{p(\cdot), u(\cdot)}\left(\ell_{q(\cdot)}\right)} .
$$

Proposition 2. Let $p, q, u \in \mathcal{P}\left(\mathbb{R}^{n}\right)$ with $p(x) \leq u(x)$ and $0<t<+\infty$.

Let $\left(f_{\nu}\right)_{\nu} \subset \mathcal{M}\left(\mathbb{R}^{n}\right)$

$$
\left\|\left(\left|f_{\nu}\right|^{t}\right)_{\nu}\right\|_{M_{\frac{p(\cdot)}{t}, \frac{u(\cdot)}{t}}\left(\ell_{\frac{q(\cdot)}{t}}\right)}=\left\|\left(f_{\nu}\right)_{\nu}\right\|_{M_{p(\cdot), u(\cdot)}\left(\ell_{q(\cdot)}\right)}
$$

with the usual modification every time $q(x)=+\infty$.

\section{-Triebel-Lizorkin-Morrey spaces.}

We first recall a Littlewood-Paley partition of unity $\left\{\psi_{\nu}\right\}, \nu \geq 0$.

The functions $\psi_{\nu}$ are defined as follows. Let $\psi_{0} \in C_{0}^{\infty}\left(\mathbb{R}^{n}\right)$ such that $\psi_{0} \equiv 1$ on $B(0 ; 1)$ and $\operatorname{supp} \psi_{0} \subset B(0 ; 2)$.

Set

$$
\psi_{\nu}(\xi)=\psi_{0}\left(2^{-\nu} \xi\right)-\psi_{0}\left(2^{-\nu+1} \xi\right) \text { for all } \nu \in \mathbb{N} .
$$

Then $\psi_{\nu}$ is supported on the dyadic shell

$$
D_{\nu}=\left\{\xi \in \mathbb{R}^{n}: 2^{\nu-1} \leq|\xi| \leq 2^{\nu+1}\right\} .
$$

If $f \in \mathcal{S}^{\prime}$, then

$$
f=\sum_{\nu \geq 0} \psi_{\nu} f
$$

The Fourier multiplier $\psi_{j}(D)$ with symbol $\psi_{j}$ is defined as

$$
\psi_{\nu}(D) f(x)=\mathcal{F}^{-1}\left(\psi_{\nu} \cdot \hat{f}\right)(x)=\int_{\mathbb{R}^{n}} \psi_{\nu}(\xi) \hat{f}(\xi) \mathrm{e}^{\mathrm{i} x \cdot \xi} \mathrm{d} \xi
$$

Definition 3. Let $\left\{\psi_{\nu}\right\}$ be the usual Littlewood-Paley partition of unity. Let $s: \mathbb{R}^{n} \rightarrow$ $\mathbb{R}, p, q \in \mathcal{P}^{\log }\left(\mathbb{R}^{n}\right)$ and $u \in \mathcal{P}\left(\mathbb{R}^{n}\right)$ such that $0<p^{-} \leq p(x) \leq u(x) \leq \sup u<+\infty$ and $q^{-}, q^{+} \in(0,+\infty)$. The Triebel-Lizorkin-Morrey spaces $\mathcal{E}_{p(\cdot), u(\cdot), q(\cdot)}^{s(\cdot)}$ consists of all distributions $f \in \mathcal{S}^{\prime}\left(\mathbb{R}^{n}\right)$ such that

$$
\|f\|_{\mathcal{E}_{p(\cdot), u(\cdot), q(\cdot)}^{s()}}:=\left\|\psi_{0}(D) f\right\|_{M_{p(\cdot), u(\cdot)}}+\left\|\left(2^{\nu s(\cdot)} \psi_{\nu}(D) f_{\nu}\right)_{\nu \geq 1}\right\|_{M_{p(\cdot), u(\cdot)}\left(\ell_{q(\cdot)}\right)}<+\infty
$$

Remark 2. [4](remark4.4) Note that $\|\cdot\|_{\mathcal{E}_{p(\cdot), u(\cdot), q(\cdot)}^{s(\cdot)}}$ defined a quasinorm on $\mathcal{E}_{p(\cdot), u(\cdot), q(\cdot)}^{s(\cdot)}$. It is a norm when $\min \left(p^{-}, q^{-}\right) \geq 1$. 


\section{Basic tools}

In this section we present some useful results for the last section. At First, we recall the $\eta$-functions defined by

$$
\eta_{\nu, m}(x)=2^{n \nu}\left(1+2^{\nu}|x|\right)^{-m}, \quad \nu \in \mathbb{N}_{0}, m>0 .
$$

Note that $\eta_{\nu, m} \in L_{1}$ for $m>n$ and the corresponding $L_{1}$-norm does not depend on $\nu$.

The following lemma is from [8](Lemma19) and [10](Lemma6.1)

Lemma 1. Let $\alpha \in C_{\text {loc }}^{\text {log }}\left(\mathbb{R}^{n}\right)$ and let $m \geq 0, R \geq c_{\text {log }}(\alpha)$, where $c_{\text {log }}$ is the constant from (1) for $\alpha$.

Then

$$
2^{\nu \alpha(x)} \eta_{\nu, m+R}(x-y) \leq c 2^{\nu \alpha(y)} \eta_{\nu, m}(x-y)
$$

with $c>0$ independent of $x, y \in \mathbb{R}^{n}$ and $\nu \in \mathbb{N}_{0}$.

The following lemma is from [10](lemma A.6).

Lemma 2. Let $t>0, \nu \in \mathbb{N}_{0}$ and $m>n$. Then there exists $c=c(t, m, n)$ such that for all $g \in \mathcal{S}^{\prime}\left(\mathbb{R}^{n}\right)$ with supp $\mathcal{F} g \subset\left\{\xi \in \mathbb{R}^{n}:|\xi| \leq 2^{\nu+1}\right\}$, We have

$$
|g(x)| \leq c\left(\eta_{\nu, m} *|g|^{t}(x)\right)^{1 / t}, \quad x \in \mathbb{R}^{n} .
$$

The following lemma is from[4](theorem3.3).

Lemma 3. Let $p, q \in \mathcal{P}^{\log }\left(\mathbb{R}^{n}\right)$ and $u \in \mathcal{P}\left(\mathbb{R}^{n}\right)$ such that $1<p^{-} \leq p(x) \leq u(x) \leq \sup u<$ $+\infty$ and $q^{-}, q^{+} \in(1,+\infty)$. If

$$
m>n+n \max \left\{0, \sup _{x \in \mathbb{R}^{n}}\left(\frac{1}{p(x)}-\frac{1}{u(x)}\right)-\frac{1}{p_{\infty}}\right\}
$$

then there exists $c>0$ such that for all sequences $\left(f_{\nu}\right)_{\nu} \subset M_{p(\cdot), u(\cdot)}\left(\ell_{q(\cdot)}\right)$.

$$
\left\|\left(\eta_{\nu, m} * f_{\nu}\right)_{\nu}\right\|_{M_{p(\cdot), u(\cdot)}\left(\ell_{q(\cdot)}\right)} \leq c\left\|\left(f_{\nu}\right)_{\nu}\right\|_{M_{p(\cdot), u(\cdot)}\left(\ell_{q(\cdot)}\right)} .
$$

The following lemma is from[1](Corollary 4.8.)

Lemma 4. Let $p \in \mathcal{P}^{\log }\left(\mathbb{R}^{n}\right)$ and $u \in \mathcal{P}$ with $1<p^{-} \leq p(x) \leq u(x) \leq \sup u<+\infty$. If $m>n+n \max \left\{0, \sup _{x \in \mathbb{R}^{n}}\left(\frac{1}{p(x)}-\frac{1}{u(x)}\right)-\frac{1}{p_{\infty}}\right\}$.

Then there exists $c>0$ such that

$$
\left\|\eta_{\nu, m} * f\right\|_{M_{p(\cdot), u(\cdot)}} \leq c\|f\|_{M_{p(\cdot), u(\cdot)}} .
$$

The following lemma is from[4](Lemma 3.7). 
Lemma 5. Let $p, u, q \in \mathcal{P}\left(\mathbb{R}^{n}\right)$ with $p(x) \leq u(x)$. Let $\delta>0$. For any sequence $\left(g_{j}\right)_{j \in \mathbb{N}_{0}}$ of non negative measurable functions on $\mathbb{R}^{n}$, we denote

$$
G_{\nu}(x):=\sum_{j=0}^{+\infty} 2^{-|\nu-j| \delta} g_{j}(x), \quad x \in \mathbb{R}^{n}, \nu \in \mathbb{N}_{0} .
$$

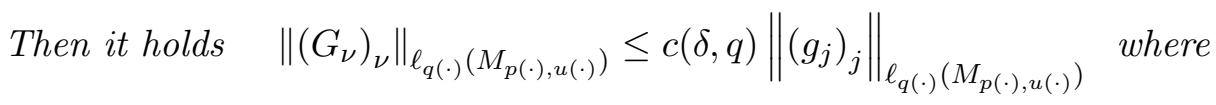

$$
c(\delta, q)=\max \left(\sum_{\nu \in \mathbb{Z}} 2^{-|\nu| \delta},\left[\sum_{\nu \in \mathbb{Z}} 2^{-|\nu| \delta q^{-}}\right]^{1 / q^{-}}\right) .
$$

\section{Boundedness of pseudo-differential operators}

We will use symbols for which $x$-regularity is measured in Hölder-Zygmund spaces.

Definition 4. [14] The function $a(x, \xi)$ on $\mathbb{R}^{n} \times \mathbb{R}^{n}$ belongs to the symbol class $C_{*}^{\ell} S_{1, \delta}^{m}, \delta \in$ $[0,1], \ell>0$ if it is smooth in $\xi$ and satisfies the following estimates:

$$
\left\{\begin{array}{l}
\left\|\partial_{\xi}^{\alpha} a(\cdot, \xi)\right\|_{C_{*}^{\ell} S_{1, \delta}^{m}} \leq c_{\alpha}\langle\xi\rangle^{m-|\alpha|+\ell \delta} \text { and } \\
\left|\partial_{\xi}^{\alpha} a(x, \xi)\right| \leq c_{\alpha}^{\prime}\langle\xi\rangle^{m-|\alpha|}
\end{array}\right.
$$

In $(5),\langle\xi\rangle$ stand for $\left(1+|\xi|^{2}\right)^{1 / 2}$.

A pseudo-differential operator on $\mathcal{E}_{p(\cdot), u(\cdot), q(\cdot)}^{s()}$ with symbol $a \in C_{*}^{\ell} S_{1, \delta}^{m}$ is defined by

$$
a(x, D) f(x)=\frac{1}{(2 \pi)^{n}} \int_{\mathbb{R}^{n}} \mathrm{e}^{\mathrm{i} x \cdot \xi} a(x, \xi) \mathcal{F} f(\xi) \mathrm{d} \xi, \quad f \in \mathcal{E}_{p(\cdot), u(\cdot), q(\cdot)}^{s(\cdot)} .
$$

Definition 5. We call elementary symbol in the class $C_{*}^{\ell} S_{1, \delta}^{m}, \delta \in[0,1], \ell>0$ an expression of the form

$$
a(x, \xi)=\sum_{j \geq 0} a_{j}(x) \psi_{j}(\xi)
$$

where $\psi_{0}$ is smooth supported on the ball $B(0,2), \psi_{j}(\xi)=\psi\left(2^{-j} \xi\right)$ and $\psi \in C_{0}^{\infty}$ is supported on the dyadic shell $D_{0}=\left\{\xi \in \mathbb{R}^{n}: 1 / 2 \leq|\xi| \leq 2\right\}$, while $a_{j}$ is uniformly bounded sequence such that

$$
\left\|a_{j}\right\|_{C_{*}^{\ell} S_{1, \delta}^{m}} \leq c 2^{j(m+\ell \delta)} .
$$

Since $a(x, D)$ and $\psi_{j}(D)$ do not commute, to study boundedness of $a(x, D)$, the symbol reduction method due to Coifman and Meyer[6] makes it possible to be limited to elementary symbols.

Therefore, the operator $a(x, D)$ with symbol $a$ can be resolved into "elementary operators" $a_{k}(x, D)$ with symbols $a_{k}$. This idea has been exploited to establish continuity of pseudo-differential operators with non-regular symbols in inhomogeneous Sobolev spaces $H^{s, p}$ and Hölder-Zygmund spaces $C_{*}^{\ell}$ ( see [12] and [2]). 
Lemma 6. [14] Let $f=\sum_{j \geq 0} f_{j}$ in $\mathcal{S}^{\prime}$, with supp $\hat{f}_{j} \subset B\left(0, A 2^{j}\right)$ for some $A>0$. Then, for $\ell>0$,

$$
\|f\|_{C_{*}^{\ell}} \leq c(A) \sup _{j \geq 0}\left\{2^{j \ell}\left\|f_{j}\right\|_{L_{\infty}}\right\} .
$$

The following lemmas plays a fundamental role in the proof of the boundedness of pseudo-differential operators on $\mathcal{E}_{p(\cdot), u(\cdot), q(\cdot)}^{s(\cdot)}$.

Lemma 7. Let $c_{1}, c_{2}>0, s \in C_{l o c}^{\text {log }}, p, q \in \mathcal{P}^{\text {log }}\left(\mathbb{R}^{n}\right)$ and $u \in \mathcal{P}\left(\mathbb{R}^{n}\right)$ such that $0<p^{-} \leq p(x) \leq u(x) \leq \sup u<\infty$ and $q^{-}, q^{+} \in(0,+\infty)$. Let $\left\{f_{k}\right\}_{k \in \mathbb{N}_{0}}$ be a sequence of tempered distributions such that

$$
\text { supp } \mathcal{F} f_{0} \subset B\left(0,2 c_{2}\right)
$$

and

$$
\operatorname{supp} \mathcal{F} f_{k} \subset\left\{\xi \in \mathbb{R}^{n}: c_{1} 2^{k-1}<|\xi|<c_{2} 2^{k+1}\right\} \text { for } k>0
$$

Then

$$
\left\|\sum_{k=0}^{+\infty} f_{k}\right\|_{\mathcal{E}_{p(\cdot), u(\cdot), q(\cdot)}^{s(\cdot)}} \lesssim\left\|\left(2^{k s(\cdot)} f_{k}\right)_{k}\right\|_{M_{p(\cdot), u(\cdot)}\left(\ell_{q(\cdot)}\right)} .
$$

Proof. Let $\left\{\psi_{j}\right\}$ be the Littlewood-Paley partition of unity defined above. By hypothesis, $\psi_{j}, j \geq 1$ are supported on the dyadic shell $D_{j}$, while $\psi_{0}$ is supported on the ball $B(0 ; 2)$. Hence, there is $N_{1}, N_{2} \in \mathbb{N}_{0}$ such that

$$
\begin{aligned}
\psi_{0}(D)\left(\sum_{k=0}^{+\infty} f_{k}\right) & =\psi_{0}(D)\left(\sum_{k=0}^{N_{1}} f_{k}\right) \\
\text { and } \psi_{j}(D)\left(\sum_{k=0}^{+\infty} f_{k}\right) & =\psi_{j}(D)\left(\sum_{k=j-N_{1}}^{j+N_{2}} f_{k}\right)
\end{aligned}
$$

Then

$$
\left\|\sum_{k=0}^{+\infty} f_{k}\right\|_{\mathcal{E}_{p(\cdot), u(\cdot), q(\cdot)}^{s(\cdot)}}=\left\|\sum_{k=0}^{N_{1}} \check{\psi}_{0} * f_{k}\right\|_{M_{p(\cdot), u(\cdot)}}+\left\|\left\{2^{j s(\cdot)} \sum_{k=j-N_{1}}^{j+N_{2}} \check{\psi}_{j} * f_{k}\right\}_{j \geq N_{1}}\right\|_{M_{p(\cdot), u(\cdot)}\left(\ell_{q(\cdot)}\right)}
$$

- Let us first estimate $\left\|\left\{2^{j s(\cdot)} \sum_{k=j-N_{1}}^{j+N_{2}} \check{\psi}_{j} * f_{k}\right\}_{j \geq 1}\right\|_{M_{p(\cdot), u(\cdot)}\left(\ell_{q(\cdot)}\right)}$

Since $\check{\psi}_{j} * f_{k} \in \mathcal{S}^{\prime}$ and $\operatorname{supp} \mathcal{F}\left(\check{\psi}_{j} * f_{k}\right) \subset\left\{\xi \in \mathbb{R}^{n}:|\xi| \leq 2^{j+1}\right\}$, then, by lemma 2 ,

$$
\left|\check{\psi}_{j} * f_{k}\right| \lesssim\left(\eta_{j, m} *\left|f_{k}\right|^{t}\right)^{1 / t}, \quad k=j-N_{1}, \ldots, j+N_{2}
$$


for any $m>n+c_{\text {log }}(s)+n \max \left\{0, \sup _{x \in \mathbb{R}^{n}}\left(\frac{1}{p(x)}-\frac{1}{u(x)}\right)-\frac{1}{p_{\infty}}\right\}$ and any $t>0$.

Thus

$\left\|\left\{2^{j s(\cdot)} \sum_{k=j-N_{1}}^{j+N_{2}} \check{\psi}_{j} * f_{k}\right\}_{j \geq N_{1}}\right\|_{M_{p(\cdot), u(\cdot)}\left(\ell_{q(\cdot)}\right)} \lesssim\left\|\left\{\sum_{k=j-N_{1}}^{j+N_{2}} 2^{j s(\cdot)}\left(\eta_{j, m} *\left|f_{k}\right|^{t}\right)^{1 / t}\right\}_{j}\right\|_{M_{p(\cdot), u(\cdot)}\left(\ell_{q(\cdot))}\right.}$

By lemma 1, we can move $2^{j s(\cdot)}$ inside the convolution

$$
2^{j s(\cdot)}\left(\eta_{j, m} *\left|f_{k}\right|^{t}\right)^{1 / t} \lesssim\left(\eta_{j, m-c_{l o g}(s)} * 2^{j s(\cdot) t}\left|f_{k}\right|^{t}\right)^{1 / t} .
$$

Then

$$
\begin{aligned}
\left\|\left\{2^{j s(\cdot)} \sum_{k=j-N_{1}}^{j+N_{2}} \check{\psi}_{j} * f_{k}\right\}_{j \geq N_{1}}\right\|_{M_{p(\cdot), u(\cdot)}\left(\ell_{q(\cdot)}\right)} \\
\quad \lesssim\left\|\left\{\sum_{k=j-N_{1}}^{j+N_{2}}\left(\eta_{j, m-c_{l o g}(s)} * 2^{j s(\cdot) t}\left|f_{k}\right|^{t}\right)^{1 / t}\right\}_{j}\right\|_{M_{p(\cdot), u(\cdot)}\left(\ell_{q(\cdot)}\right)} \\
=\left\|\left\{\sum_{k=j-N_{1}}^{j+N_{2}}\left(\eta_{j, m-c_{\log }(s)} * 2^{j s(\cdot) t}\left|f_{k}\right|^{t}\right)\right\}_{j}\right\|_{M_{\frac{p(\cdot)}{t}, \frac{u(\cdot)}{t}}\left(\ell_{\frac{q(\cdot)}{t}}\right)}
\end{aligned}
$$

With $t \in\left(0, \min \left\{1, p^{-}, q^{-}\right\}\right)$, lemma 4 yields

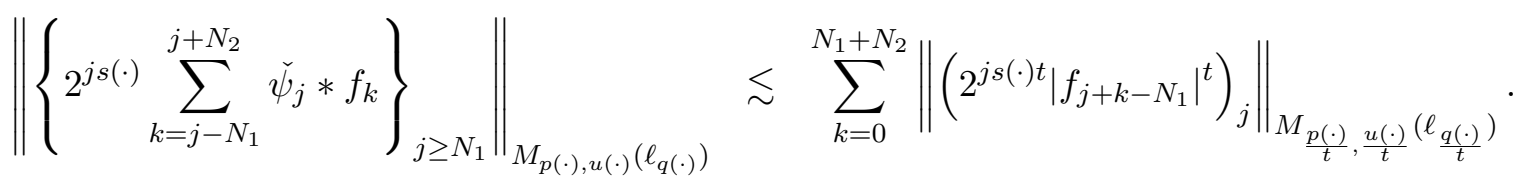

$$
\begin{aligned}
& \text { Then }\left\|\left\{2^{j s(\cdot)} \sum_{k=j-N_{1}}^{j+N_{2}} \check{\psi}_{j} * f_{k}\right\}_{j \geq 1}\right\|_{M_{p(\cdot), u(\cdot)}\left(\ell_{q(\cdot)}\right)} \lesssim\left\|\left(2^{k s(\cdot)} f_{k}\right)_{k}\right\|_{M_{p(\cdot), u(\cdot)}\left(\ell_{q(\cdot)}\right)} .
\end{aligned}
$$

- Now we estimate the first term .

Since $\operatorname{supp} \mathcal{F}\left(\check{\psi}_{0} * f_{k}\right) \subset\left\{\xi \in \mathbb{R}^{n}:|\xi| \leq 2\right\}$, then by lemma $2,\left|\check{\psi}_{0} * f_{k}\right| \lesssim\left|f_{k}\right|$.

Thus

$$
\begin{aligned}
\left\|\sum_{k=0}^{N_{1}} \check{\psi}_{0} * f_{k}\right\|_{M_{p(\cdot), u(\cdot)}} & \lesssim \sum_{k=0}^{N_{1}}\left\|f_{k}\right\|_{M_{p(\cdot), u(\cdot)}} \\
& =\sum_{k=0}^{N_{1}}\left\|\left(0, \ldots, f_{k}, 0, \ldots\right)\right\|_{\ell_{q(\cdot)}\left(M_{p(\cdot), u(\cdot)}\right)}
\end{aligned}
$$




$$
\lesssim\left\|\left(2^{k s(\cdot)} f_{k}\right)_{k}\right\|_{\ell_{q(\cdot)}\left(M_{p(\cdot), u(\cdot)}\right)}
$$

The proof is completed.

Lemma 8. Let $c>0, s \in C_{\text {loc }}^{\log }, p, q \in \mathcal{P}^{\log }\left(\mathbb{R}^{n}\right)$ and $u \in \mathcal{P}\left(\mathbb{R}^{n}\right)$ such that $0<p^{-} \leq p(x) \leq u(x) \leq \sup u<+\infty, s^{-}>0$ and $q^{-}, q^{+} \in(0,+\infty)$. Let $\left\{f_{k}\right\}_{k \in \mathbb{N}_{0}}$ be $a$ sequence of tempered distributions such that

$$
\operatorname{supp} \mathcal{F} f_{k} \subset B\left(0, c 2^{k+1}\right)
$$

Then

$$
\left\|\sum_{k=0}^{+\infty} f_{k}\right\|_{\mathcal{E}_{p(\cdot), u(\cdot), q(\cdot)}^{s()}} \lesssim\left\|\left(2^{k s(\cdot)} f_{k}\right)_{k}\right\|_{M_{p(\cdot), u(\cdot)}\left(\ell_{q(\cdot)}\right)}
$$

Proof. In view of the hypothesis on $\operatorname{Supp} \psi_{j}$, there is $N \in \mathbb{N}_{0}$ such that

$$
\left\|\sum_{k=0}^{+\infty} f_{k}\right\|_{\mathcal{E}_{p(\cdot), u(\cdot), q(\cdot)}^{s()}}=\left\|\psi_{0}(D)\left(\sum_{k=0}^{+\infty} f_{k}\right)\right\|_{M_{p(\cdot), u(\cdot)}}+\left\|\left\{2^{j s(\cdot)} \psi_{j}(D)\left(\sum_{k=j-N}^{+\infty} f_{k}\right)\right\}_{j \geq N}\right\|_{M_{p(\cdot), u(\cdot)}\left(\ell_{q(\cdot)}\right)} .
$$

(i) At first we estimate $\left\|\left\{2^{j s(\cdot)} \psi_{j}(D)\left(\sum_{k=j-N}^{+\infty} f_{k}\right)\right\}_{j}\right\|_{M_{p(\cdot), u(\cdot)}\left(\ell_{q(\cdot)}\right)}$.

We have

$$
\left\|\left\{2^{j s(\cdot)} \psi_{j}(D)\left(\sum_{k=j-N}^{+\infty} f_{k}\right)\right\}_{j \geq N}\right\|_{\ell_{q(\cdot)}\left(M_{p(\cdot), u(\cdot)}\right)}=\left\|\left\{\sum_{k=j-N}^{+\infty} 2^{j s(\cdot)}\left(\check{\psi}_{j} * f_{k}\right)\right\}_{j}\right\|_{M_{p(\cdot), u(\cdot)}\left(\ell_{q(\cdot)}\right)}
$$

Since

$$
\left\{\begin{array}{l}
\operatorname{supp} \mathcal{F}\left(\check{\psi}_{j} * f_{k}\right) \subset\left\{\xi \in \mathbb{R}^{n}:|\xi| \leq 2^{j+1}\right\} \\
\operatorname{supp} \mathcal{F}\left(\check{\psi}_{j} * f_{k}\right) \subset\left\{\xi \in \mathbb{R}^{n}:|\xi| \leq 2^{k+1}\right\}
\end{array}\right.
$$

by lemma 2 ,

$$
\left\{\begin{array}{l}
2^{j s(\cdot)}\left(\check{\psi}_{j} * f_{k}\right) \lesssim 2^{j s(\cdot)}\left(\eta_{j, m} *\left|f_{k}\right|^{t}\right)^{1 / t} \\
2^{j s(\cdot)}\left(\check{\psi}_{j} * f_{k}\right) \lesssim 2^{j s(\cdot)}\left(\eta_{k, m} *\left|f_{k}\right|^{t}\right)^{1 / t}
\end{array}\right.
$$

for $m>n+c_{l o g}(1 / q)+c_{l o g}(s)+n \max \left\{0, \sup _{x \in \mathbb{R}^{n}}\left(\frac{1}{p(x)}-\frac{1}{u(x)}\right)-\frac{1}{p_{\infty}}\right\}$ and $t>0$.

Therefore

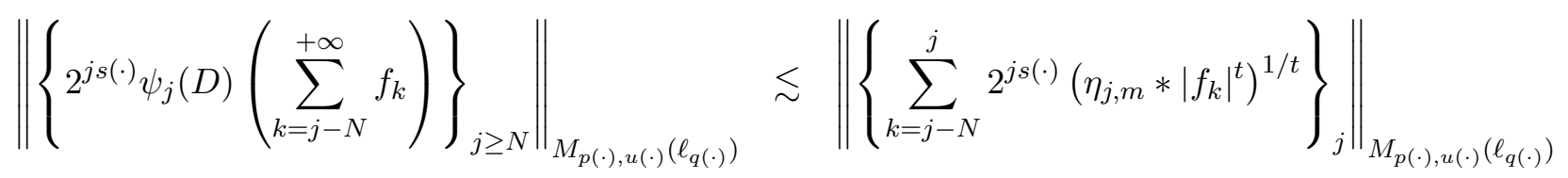




$$
+\left\|\left\{\sum_{k=j+1}^{+\infty} 2^{-(k-j) s(\cdot)} 2^{k s(\cdot)}\left(\eta_{k, m} *\left|f_{k}\right|^{t}\right)^{1 / t}\right\}_{j}\right\|_{M_{p(\cdot), u(\cdot)}\left(\ell_{q(\cdot)}\right)}
$$

Let us estimate each one of the two terms on the right-hand side.

Using lemmas 1 we can move $2^{\nu s(\cdot)}$ inside the convolution $2^{\nu s(\cdot)}\left(\eta_{\nu, m} *\left|f_{k}\right|^{t}\right)^{1 / t}$. And we have

$$
2^{\nu s(\cdot)}\left(\eta_{\nu, m} *\left|f_{k}\right|^{t}\right)^{1 / t} \lesssim\left(\eta_{\nu, m_{0}} * 2^{\nu s(\cdot) t}\left|f_{k}\right|^{t}\right)^{1 / t}, \nu=j \text { or } k \text { where } m_{0}=m-c_{l o g}(s)
$$

Thus

$$
\text { • } \begin{aligned}
\left\|\left\{\sum_{k=j-N}^{j} 2^{j s(\cdot)}\left(\eta_{j, m} *\left|f_{k}\right|^{t}\right)^{1 / t}\right\}_{j}\right\|_{M_{p(\cdot), u(\cdot)}\left(\ell_{q(\cdot)}\right)} & =\left\|\left\{\sum_{k=j-N}^{j} \eta_{j, m_{0}} * 2^{j s(\cdot) t}\left|f_{k}\right|^{t}\right\}_{j}\right\|_{M_{\frac{p(\cdot)}{t}, \frac{u(\cdot)}{t}\left(\ell_{\frac{q(\cdot)}{t}}\right)}} \\
& \lesssim \sum_{k=-N}^{0}\left\|\left\{\eta_{j, m_{0}} * 2^{j s(\cdot) t}\left|f_{k+j}\right|^{t}\right\}_{j}\right\|_{M_{\frac{p(\cdot)}{t}, \frac{u(\cdot)}{t}}\left(\ell_{\frac{q(\cdot)}{t}}\right)} .
\end{aligned}
$$

For $t \in\left(0, \min \left\{p^{-}, q^{-}\right\}\right)$, lemma 3 yields

$$
\sum_{k=-N}^{0}\left\|\left\{\eta_{j, m_{0}} * 2^{j s(\cdot) t}\left|f_{k+j}\right|^{t}\right\}_{j}\right\|_{M_{\frac{p(\cdot)}{t}, \frac{u(\cdot)}{t}}\left(\ell_{\frac{q(\cdot)}{t}}\right.} \lesssim \sum_{k=-N}^{0}\left\|\left\{2^{j s(\cdot) t}\left|f_{k+j}\right|^{t}\right\}_{j}\right\|_{M_{\frac{p(\cdot)}{t}, \frac{u(\cdot)}{t}}\left(\ell_{\frac{q(\cdot)}{t}}\right)} .
$$

Then

$$
\left\|\left\{\sum_{k=j-N}^{j} 2^{j s(\cdot)}\left(\eta_{j, m} *\left|f_{k}\right|^{t}\right)^{1 / t}\right\}_{j}\right\|_{M_{p(\cdot), u(\cdot)}\left(\ell_{q(\cdot)}\right)} \lesssim\left\|\left(2^{j s(\cdot)} f_{j}\right)_{j}\right\|_{M_{p(\cdot), u(\cdot)}\left(\ell_{q(\cdot)}\right)} .
$$

And

- $\left\|\left\{\sum_{k=j+1}^{+\infty} 2^{-(k-j) s(\cdot)} 2^{k s(\cdot)}\left(\eta_{k, m} *\left|f_{k}\right|^{t}\right)^{1 / t}\right\}_{j}\right\|_{M_{p(\cdot), u(\cdot)}\left(\ell_{q(\cdot)}\right)}$

$$
\begin{aligned}
& \lesssim\left\|\left\{\sum_{k=j+1}^{+\infty} 2^{-|j-k| s(\cdot)}\left(\eta_{k, m_{0}} * 2^{k s(\cdot) t}\left|f_{k}\right|^{t}\right)\right\}_{j}\right\|_{M_{\frac{p(\cdot)}{t}, \frac{u(\cdot)}{t}}\left(\ell_{\left.\frac{q(\cdot)}{t}\right)}\right.} \\
& \lesssim\left\|\left\{\sum_{k=j+1}^{+\infty} 2^{-|j-k| s^{-}}\left(\eta_{k, m_{0}} * 2^{k s(\cdot) t}\left|f_{k}\right|^{t}\right)\right\}_{j}\right\|_{M_{\frac{p(\cdot)}{t}, \frac{u(\cdot)}{t}}\left(\ell_{\left.\frac{q(\cdot)}{t}\right)}\right.}
\end{aligned}
$$


M. Congo, M. F. Ouedraogo / Eur. J. Pure Appl. Math, 15 (1) (2022), 47-63

$$
\lesssim\left\|\left\{\sum_{k=0}^{+\infty} 2^{-|j-k| s^{-}}\left(\eta_{k, m_{0}} * 2^{k s(\cdot) t}\left|f_{k}\right|^{t}\right)\right\}_{j}\right\|_{M_{\frac{p(\cdot)}{t}, \frac{u(\cdot)}{t}} \ell_{\frac{q(\cdot)}{t}}}
$$

By lemma 5 ,

$\left\|\left\{\sum_{k=0}^{+\infty} 2^{-|j-k| s^{-}}\left(\eta_{k, m_{0}} * 2^{k s(\cdot) t}\left|f_{k}\right|^{t}\right)\right\}_{j}\right\|_{M_{\frac{p(\cdot)}{t}, \frac{u(\cdot)}{t}}\left(\ell_{\frac{q(\cdot)}{t}}\right)} \lesssim\left\|\left(\eta_{k, m_{0}} * 2^{k s(\cdot) t}\left|f_{k}\right|^{t}\right)_{k}\right\|_{M_{\frac{p(\cdot)}{t}, \frac{u(\cdot)}{t}}\left(\ell_{\frac{q(\cdot)}{t}}\right)}$.

For $t \in\left(0, \min p^{-}, q^{-}\right)$, lemma 3 yields

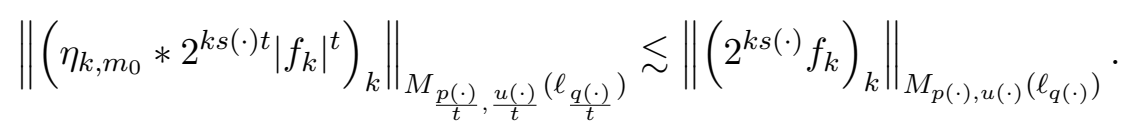

Then

$$
\left\|\left\{\sum_{k=j+1}^{+\infty} 2^{-(k-j) s(\cdot)} 2^{k s(\cdot)}\left(\eta_{k, m_{0}} *\left|f_{k}\right|^{t}\right)^{1 / t}\right\}_{j}\right\|_{M_{p(\cdot), u(\cdot)}\left(\ell_{q(\cdot)}\right)} \lesssim\left\|\left(2^{k s(\cdot)} f_{k}\right)_{k}\right\|_{M_{p(\cdot), u(\cdot)}\left(\ell_{q(\cdot)}\right)} .
$$

(ii) Now we estimate $\left\|\psi_{0}(D)\left(\sum_{k=0}^{+\infty} f_{k}\right)\right\|_{M_{p(\cdot), u(\cdot)}}$.

Since

$$
\operatorname{supp} \mathcal{F}\left(\check{\psi}_{0} * f_{k}\right) \subset\left\{\xi \in \mathbb{R}^{n}:|\xi| \leq 2^{k+1}\right\}
$$

Then

$$
\begin{aligned}
\left\|\psi_{0}(D)\left(\sum_{k=0}^{+\infty} f_{k}\right)\right\|_{M_{p(\cdot), u(\cdot)}} & =\left\|\sum_{k=0}^{N} \psi_{0} * f_{k}\right\|_{M_{p(\cdot), u(\cdot)}} \\
& \lesssim\left\|\sum_{k=0}^{\infty}\left(\eta_{k, m} *\left|f_{k}\right|^{t}\right)^{1 / t}\right\|_{M_{p(\cdot), u(\cdot)}},
\end{aligned}
$$

for $m>n+c_{l o g}(1 / q)+c_{l o g}(s)+n \max \left\{0, \sup _{x \in \mathbb{R}^{n}}\left(\frac{1}{p(x)}-\frac{1}{u(x)}\right)-\frac{1}{p_{\infty}}\right\}$ by lemma 2 .

Then by lemma 1

$$
\begin{aligned}
\left\|\psi_{0}(D)\left(\sum_{k=0}^{+\infty} f_{k}\right)\right\|_{M_{p(\cdot), u(\cdot)}} & \lesssim\left\|\sum_{k=0}^{\infty} 2^{-k s^{-}}\left(\eta_{k, m-c_{l o g}(s)} * 2^{k s(\cdot) t}\left|f_{k}\right|^{t}\right)\right\|_{M_{\frac{p(\cdot)}{t}, \frac{u(\cdot)}{t}}} \\
= & \left\|\left\{\sum_{k=0}^{+\infty} 2^{-k s^{-}}\left(\eta_{k, m-c_{l o g}(s)} * 2^{k s(\cdot) t}\left|f_{k}\right|^{t}\right)\right\}_{j}\right\|_{M_{\frac{p(\cdot)}{t}, \frac{u(\cdot)}{t}}\left(\ell_{\left.\frac{q(\cdot)}{t}\right)}\right.}
\end{aligned}
$$


Thus

$$
\begin{aligned}
\left\|\psi_{0}(D)\left(\sum_{k=0}^{+\infty} f_{k}\right)\right\|_{M_{p(\cdot), u(\cdot)}} & \lesssim\left\|\left(\eta_{k, m-c_{l o g}(s)} * 2^{k s(\cdot) t}\left|f_{k}\right|^{t}\right)_{k}\right\|_{M_{\frac{p(\cdot)}{t}, \frac{u(\cdot)}{t}}\left(\ell_{\left.\frac{q(\cdot)}{t}\right)}\right.} \\
& \lesssim\left\|\left(2^{k s(\cdot)} f_{k}\right)_{k}\right\|_{M_{p(\cdot), u(\cdot)}\left(\ell_{q(\cdot)}\right)} \quad \text { by lemma } 5 \text { and lemma } 2 .
\end{aligned}
$$

The proof is completed.

Theorem 1. Let $a(x, \xi) \in C_{*}^{\ell} S_{1, \delta}^{m}$ where $m \in \mathbb{R}, \delta \in[0,1]$ and $\ell>0$. Let $1 \leq p^{-} \leq p(x) \leq$ $u(x) \leq \sup u<+\infty$ and $q^{-}, q^{+} \in[1,+\infty)$. Let $s \in C_{\text {loc }}^{\text {log }}$ such that $0<s^{-} \leq s^{+}<\ell$. Then

$$
a(x, D): \mathcal{E}_{p(\cdot), u() \cdot,(\cdot)}^{s(\cdot)} \longrightarrow \mathcal{E}_{p(\cdot), u(\cdot), q(\cdot)}^{s(\cdot)}
$$

is bounded.

Proof. We recall that the symbol reduction method, due to Coifman and Meyer[6], makes it possible to be limited to symbols $a(x, \xi) \in C_{*}^{\ell} S_{1, \delta}^{m}$ of the form (see [14] and [2])

$$
a(x, \xi)=\sum_{j \geq 0} \sigma_{j}(x) \psi_{j}(\xi)
$$

where $\sigma_{j}$ satisfies

$$
\begin{aligned}
& \left\|\sigma_{j}\right\|_{C_{*}^{\ell}} \leq c 2^{j(m+\ell \delta)} \\
& \text { and }\left\|\sigma_{j}\right\|_{L_{\infty}} \leq c
\end{aligned}
$$

with $c$ depending on $\delta$ and $\ell$ but not on $j$. And $\psi_{j}$ is exactly a Littlewood-Paley function. We have

$$
\begin{aligned}
\sigma_{j}(x) & =\sum_{k=0}^{+\infty} \psi_{j}(D) \sigma_{j}(x) . \\
\text { Then } \sigma_{j}(x) \psi_{j}(\xi) & =\left(\sum_{k=0}^{+\infty} \psi_{j}(D) \sigma_{j}(x) \psi_{j}(\xi)\right) . \\
\text { Therefore } a(x, \xi) & =\sum_{j=0}^{+\infty}\left(\sum_{k=0}^{+\infty} \psi_{k}(D) \sigma_{j}(x)\right) \psi_{j}(\xi) .
\end{aligned}
$$

Set $\quad a_{k j}=\psi_{k}(D) \sigma_{j}$. Then

$$
a(x, \xi)=\sum_{j=0}^{+\infty}\left(\sum_{k=0}^{+\infty} a_{k j}\right) \psi_{j}(\xi) .
$$


(i) At first, it's necessary to estimate $\left\|a_{k j}\right\|_{L_{\infty}}$.

We recall the quasinorm of $C_{*}^{\ell}:\left\|\psi_{k}(D) \sigma_{j}\right\|_{C_{*}^{\ell}}=\sup _{k} 2^{k \ell}\left\|\psi_{k}(D) \sigma_{j}\right\|_{L_{\infty}}$.

Since $\left\|\psi_{k}(D) \sigma_{j}\right\|_{C_{*}^{\ell}} \leq c\left\|\sigma_{j}\right\|_{C_{*}^{\ell}}$.

Then $\sup _{k} 2^{k \ell}\left\|\psi_{k}(D) \sigma_{j}\right\|_{L_{\infty}} \leq c\left\|\sigma_{j}\right\|_{C_{*}^{\ell}}$.

Using (9), we obtain

$$
\left\|a_{k j}\right\|_{L_{\infty}} \leq c 2^{j(m+\ell \delta)} 2^{-k \ell} .
$$

Note that $(1-\Delta)^{\frac{m}{2}}, m \in \mathbb{R}$ is an isomorphism that composes well with pseudodifferential operators (see[14] and [15]). Therefore, it is enough to examine the case $m=0$. If $m=0$ then

$$
\left\|a_{k j}\right\|_{L_{\infty}} \leq c 2^{j \ell \delta} 2^{-k \ell}
$$

(ii) Now we rewrite the symbol as a sum of three parts

$$
\begin{aligned}
a(x, \xi) & =\sum_{j \geq 0}\left(\sum_{k=0}^{j-4} a_{k j}(x)+\sum_{k=j-3}^{j+3} a_{k j}(x)+\sum_{k=j+4}^{\infty} a_{k j}(x)\right) \psi_{j}(\xi) \\
& =a_{1}(x, \xi)+a_{2}(x, \xi)+a_{3}(x, \xi)
\end{aligned}
$$

where

$$
\begin{aligned}
& a_{1}(x, D) f=\sum_{j=0}^{+\infty}\left(\sum_{k=0}^{j-4} a_{k j} \psi_{j}(D) f\right), \\
& a_{2}(x, D) f=\sum_{j=0}^{+\infty}\left(\sum_{k=j-3}^{j+3} a_{k j} \psi_{j}(D) f\right), \\
& a_{3}(x, D) f=\sum_{j=0}^{+\infty}\left(\sum_{k=j+4}^{\infty} a_{k j} \psi_{j}(D) f\right) .
\end{aligned}
$$

-We have

$$
\begin{aligned}
\mathcal{F}\left(\sum_{k=0}^{j-4} a_{k j} f_{j}\right) & =\sum_{k=0}^{j-4} \mathcal{F}\left(\psi_{k}(D) \sigma_{j}\right) * \mathcal{F}\left(\psi_{j}(D) f\right) \\
& =\sum_{k=0}^{j-4}\left(\psi_{k} \mathcal{F} \sigma_{j}\right) *\left(\psi_{j} \mathcal{F} f\right)
\end{aligned}
$$

Using the fact that $\operatorname{supp}(f * g) \subset \operatorname{supp} f+\operatorname{supp} g$ for all compactly supported distributions $f, g \in \mathcal{S}^{\prime}$, we have $\operatorname{supp} \mathcal{F}\left(\sum_{k=0}^{j-4} a_{k j} f_{j}\right) \subset\left\{\xi \in \mathbb{R}^{n}: c_{1} 2^{j-1} \leq|\xi| \leq c_{2} 2^{j+1}\right\}$ with $c_{1}, c_{2}>0$. Then lemma 7 yields 


$$
\begin{aligned}
\left\|a_{1}(x, D) f\right\|_{\mathcal{E}_{p(\cdot), u(\cdot), q(\cdot)}^{s(\cdot)}}=\left\|\sum_{j=0}^{+\infty}\left(\sum_{k=0}^{j-4} a_{k j} \psi_{j}(D) f\right)\right\|_{\mathcal{E}_{p(\cdot), u(\cdot), q(\cdot)}^{s(\cdot)}} \\
\lesssim\left\|\left(2^{j s(\cdot)} \sum_{k=0}^{j-4} a_{k j} \psi_{j}(D) f\right)_{j}\right\|_{M_{p(\cdot), u(\cdot)}\left(\ell_{q(\cdot))}\right.} \\
\lesssim\left\|\left(\sum_{k=0}^{j-4}\left\|\sigma_{j}\right\|_{L_{\infty}} 2^{j s(\cdot)} \psi_{j}(D) f\right)_{j}\right\|_{M_{p(\cdot), u(\cdot)}\left(\ell_{q(\cdot)}\right)} \\
\lesssim\left\|\left(2^{j s(\cdot)} \psi_{j}(D) f\right)_{j}\right\|_{M_{p(\cdot), u(\cdot)}\left(\ell_{q(\cdot)}\right)} \cdot
\end{aligned}
$$

Then

$$
\left\|a_{1}(x, D) f\right\|_{\mathcal{E}_{p(\cdot), u(\cdot), q(\cdot)}^{s()}} \lesssim\|f\|_{\mathcal{E}_{p(\cdot), u(\cdot), q(\cdot)}^{s(\cdot)}} .
$$

-For the second part $\left\|a_{2}(x, D) f\right\|_{\mathcal{E}_{p(\cdot), u(\cdot), q(\cdot)}^{s(\cdot)}}=\left\|\sum_{j=0}^{+\infty}\left(\sum_{k=j-3}^{j+3} a_{k j} f_{j}\right)\right\|_{\mathcal{E}_{p(\cdot), u(\cdot), q(\cdot)}^{s()}}$,

we observe that

$$
\begin{aligned}
\mathcal{F}\left(\sum_{k=j-3}^{j+3} a_{k j} f_{j}\right) & =\sum_{k=j-3}^{j+3} \mathcal{F}\left(\psi_{k}(D) \sigma_{j}\right) * \mathcal{F}\left(\psi_{j}(D) f\right) \\
& =\sum_{k=j-3}^{j+3}\left(\psi_{k} \mathcal{F} \sigma_{j}\right) *\left(\psi_{j} \mathcal{F} f\right) .
\end{aligned}
$$

Then $\mathcal{F}\left(\sum_{k=j-3}^{j+3} a_{k j} f_{j}\right)$ is supported on the ball $B\left(0,2^{j+4}\right)$.

By lemma 8,

$$
\begin{aligned}
\left\|a_{2}(x, D) f\right\|_{\mathcal{E}_{p(\cdot), u(\cdot), q(\cdot)}^{s(\cdot)}} \lesssim\left\|\left(2^{j s(\cdot)} \sum_{k=j-3}^{j+3} a_{k j} f_{j}\right)\right\|_{j} \|_{M_{p(\cdot), u(\cdot)}\left(\ell_{q(\cdot)}\right)} \\
\leq 2^{-m}\left\|\left(\sum_{k=j-3}^{j+3}\left\|a_{k j}\right\|_{L_{\infty}} 2^{j s(\cdot)} \psi_{j}(D) f\right)\right\|_{j} \|_{M_{p(\cdot), u(\cdot)}\left(\ell_{q(\cdot)}\right)} .
\end{aligned}
$$




$$
\text { One have } \sum_{k=j-3}^{j+3}\left\|a_{k j}\right\|_{L_{\infty}} \lesssim \sum_{k=-3}^{3} 2^{-k \ell}<+\infty \quad(\text { with } \delta=1) \text {. }
$$

Then

$$
\begin{aligned}
\left\|a_{2}(x, D) f\right\|_{\mathcal{E}_{p(\cdot), u(\cdot), q(\cdot)}^{s(\cdot)}} & \lesssim\left\|\left(2^{j s(\cdot)} \psi_{j}(D) f\right)_{j}\right\|_{M_{p(\cdot), u(\cdot)}\left(\ell_{q(\cdot)}\right)} \\
& \lesssim\|f\|_{\mathcal{E}_{p(\cdot), u(\cdot), q(\cdot)}^{s(\cdot)}} .
\end{aligned}
$$

- Now let us estimate last part. Since $\mathcal{F}\left(\sum_{k=j+4}^{+\infty} a_{k j} f_{j}\right)$ is not supported on any ball or shell, we cannot directly use neither lemma7 nor lemma8.

However, in $\mathcal{S}^{\prime}$ we can write

$$
\sum_{j=0}^{+\infty} \sum_{k=j+4}^{+\infty} a_{k j} f_{j}=\sum_{k=4}^{+\infty} \sum_{j=0}^{k-4} a_{k j} f_{j}
$$

We have

$$
\mathcal{F}\left(\sum_{j=0}^{k-4} a_{k j} f_{j}\right)=\sum_{j=0}^{k-4}\left(\psi_{k} \mathcal{F} a_{j}\right) *\left(\psi_{j} \mathcal{F} f\right) .
$$

We have supp $\mathcal{F}\left(\sum_{k=0}^{j-4} a_{k j} f_{j}\right) \subset\left\{\xi \in \mathbb{R}^{n}\left|c_{1} 2^{j-1} \leq\right| \xi \mid \leq c_{2} 2^{j+1}\right\}$ with $c_{1}, c_{2}>0$.

Thus we can use lemma 7.

$$
\begin{aligned}
\left\|a_{3}(x, D) f\right\|_{\mathcal{E}_{p(\cdot), u(\cdot), q(\cdot)}^{s(\cdot)}} & =\left\|\sum_{k=4}^{+\infty}\left(\sum_{j=0}^{k-4} a_{k j} f_{j}\right)\right\|_{\mathcal{E}_{p(\cdot), u(\cdot), q(\cdot)}^{s(\cdot)}} \\
& \lesssim\left\|\left(2^{k s(\cdot)} \sum_{j=0}^{k-4} a_{k j} f_{j}\right)_{k}\right\|_{M_{p(\cdot), u(\cdot)}\left(\ell_{q(\cdot)}\right)} \\
& \lesssim\left\|\left(\sum_{j=0}^{k-4}\left\|a_{k j}\right\|_{L_{\infty}} 2^{k s(\cdot)} \psi_{j}(D) f\right)_{k}\right\|_{M_{p(\cdot), u(\cdot)}\left(\ell_{q(\cdot)}\right)}
\end{aligned}
$$

If we use(13) with $\delta=1$, we have

$$
\left\|a_{3}(x, D) f\right\|_{\mathcal{E}_{p(\cdot), u(\cdot), q(\cdot)}^{s(\cdot)}} \lesssim\left\|\left(\sum_{j=0}^{k-4} 2^{j \ell} 2^{-k \ell} 2^{k s(\cdot)} \psi_{j}(D) f\right)_{k}\right\|_{M_{p(\cdot), u(\cdot)}\left(\ell_{q(\cdot)}\right)}
$$




$$
\begin{aligned}
& =\left\|\left(\sum_{j=0}^{k-4} 2^{(k-j)(s(\cdot)-\ell)} 2^{j s(\cdot)} \psi_{j}(D) f\right)_{k}\right\|_{M_{p(\cdot), u(\cdot)}\left(\ell_{q(\cdot)}\right)} \\
& \leq\left\|\left(\sum_{j=0}^{k-4} 2^{-\left|k-j \| s^{-}-\ell\right|} 2^{j s(\cdot)} \psi_{j}(D) f\right)_{k}\right\|_{M_{p(\cdot), u(\cdot)}\left(\ell_{q(\cdot)}\right)} \\
& \leq\left\|\left(\sum_{j=0}^{+\infty} 2^{-\left|k-j \| s^{-}-\ell\right|} 2^{j s(\cdot)} \psi_{j}(D) f\right)_{k}\right\|_{M_{p(\cdot), u(\cdot)}\left(\ell_{q(\cdot)}\right)}
\end{aligned}
$$

By hypothesis $\left|s^{-}-\ell\right|>0$. Therefore, by lemma 5

$$
\left\|\left(\sum_{j=0}^{k-4} 2^{-\left|k-j \| s^{-}-\ell\right|} 2^{j s(\cdot)} \psi_{j}(D) f\right)_{j}\right\|_{M_{p(\cdot), u(\cdot)}\left(\ell_{q(\cdot)}\right)} \lesssim\left\|\left(2^{j s(\cdot)} \psi_{j}(D) f\right)_{k}\right\|_{M_{p(\cdot), u(\cdot)}\left(\ell_{q(\cdot)}\right)} .
$$

Then

$$
\left\|a_{3}(x, D) f\right\|_{\mathcal{E}_{p(\cdot), u(\cdot), q(\cdot)}^{s(\cdot)}} \lesssim\|f\|_{\mathcal{E}_{p(\cdot), u(\cdot), q(\cdot)}^{s(\cdot)}} .
$$

The proof is completed.

\section{References}

[1] A. Almeida and A. Caetano. Variable exponent besov-morrey spaces. Fourier Anal. Appl., 26(5), 2020.

[2] G. Bourdaud. Une algèbre maximale d'opérateurs pseudo-différentiels. Comm. Partial Differential Equations, 13(9):1059-1083, 1988.

[3] A. Caetano and H. Kempka. Besov spaces with variable smoothness and integrability. Mathematical. Anal. and appl., 484, 2020.

[4] A. Caetano and H. Kempka. Variable exponent triebel-lizorkin-morrey spaces. Math. Anal. Appl., 484(123712), 2020.

[5] A. Caetano and H. Kempka. Decompositions with atoms and molecules for variable exponent triebel-lizorkin-morrey spaces. Constructive Approximation, 53:201-234, 2021.

[6] R. Coifman and Y. Meyer. Au delà des opérateurs pseudo-différentiels. 1978.

[7] D. Cruz-Uribe and A. Fiorenza. Variable Lebesgue Spaces. Birkhäuser, Basel, 2013. 
[8] H. Kempka and J. Vybíral. Spaces of variable smoothness and integrability: Characterizations by local means and ball means of differences. Fourier Anal. Appl., 18(4):852-891, 2012.

[9] H. Kozono and M. Yamazaki. Semilinear heat equations and the navier-stokes equation with distributions in new function spaces as initial data. Comm. Partial Differential Equations, 19:959-1014, 1994.

[10] P. Hästö L. Diening and S. Roudenko. Function spaces of variable smoothness and integrability. Funct. Anal., 256(6):1731-1768, 2009.

[11] P. Hästö L. Diening, P. Harjulehto and M. Ruzicka. Lebesgue and Sobolev Spaces with Variable Exponents., volume 2017. Springer-Verlag, Berlin, 2011.

[12] J. Marschall. Pseudodifferential operators with coefficients in sobolev spaces. Trans. Amer. Math. Soc., 307(1):335-361, 1988.

[13] J. Marschall. Nonregular pseudo-differential operators. Z. Anal. Anwend, 15(1):109$148,1996$.

[14] A. Mazzucato. Besov-morrey spaces: Function space theory and applications to nonlinear pde. Trans. Amer. Math. Soc., 355:1297-1364, 2003.

[15] Y. Sawano. A note on besov-morrey spaces and triebel-lizorkin-morrey spaces. Acta Math. Sin., 25:1223-1242, 2009.

[16] M. E. Taylor. Pseudodifferential operators and nonlinear PDE. Progress in Mathematics 100, Birkhäuser, Boston, MA,, 1991.

[17] H. Triebel. Besov spaces with variable smoothness and integrability. Birkhauser Verlag, Basel and al., 1983.

[18] W. Sickel W. Yuan and D. Yang. Morrey and campanato meet besov, lizorkin and triebel. 2005, 2010. 